\title{
Suicidal Behavior in Prodromal Huntington Disease
}

\author{
Jess G. Fiedorowicz ${ }^{a}$ b James A. Mills ${ }^{a}$ Adam Ruggle ${ }^{a}$ Douglas Langbehn ${ }^{a}$ \\ Jane S. Paulsen ${ }^{a, c}$, d PREDICT-HD Investigators of the Huntington Study Group \\ Departments of a Psychiatry, Carver College of Medicine, ${ }^{b}$ Epidemiology, College of Public Health, \\ 'Neurology, Carver College of Medicine, and d Psychology, College of Liberal Arts and Sciences, University of lowa, \\ lowa City, lowa, USA
}

\section{Key Words}

Attempted suicide $\cdot$ Cohort study $\cdot$ Huntington disease $\cdot$

Major depressive disorder $\cdot$ Risk factors $\cdot$ Suicide

\begin{abstract}
Background: Several studies have suggested a greater risk of suicide in Huntington disease (HD); however, unique risk factors for suicide in HD are not established. Objective: We sought to determine risk factors for suicidal behavior, defined as suicide or attempted suicide, in prodromal HD. Methods: From the prospective PREDICT-HD cohort, we identified 735 cases with HD gene expansion but no manifest symptoms of HD and 194 non-gene-expanded controls. In survival analysis, a number of potential risk factors for suicidal behavior were assessed, including symptoms of depression, hopelessness, substance abuse, marital status, gender, and psychiatric history. Results: During a mean of 3.7 years of prospective follow-up, 12 cases (1.6\%) attempted suicide and 1 completed suicide ( $0.1 \%)$. No suicides were observed among controls. In univariate Cox proportional hazards regression models, a history of suicide attempts (HR 8.5, $95 \% \mathrm{Cl} 2.8-26.1, \mathrm{p}<0.0002)$ and a Beck Depression Inventory II score $>13$ (HR 7.2, 95\% Cl 2.3-22.0, $\mathrm{p}<0.0006)$ were associated with suicidal behavior. These risk factors had independent effects in multivariate models. A history of incar-
\end{abstract}

ceration in the past 2 years was also associated (HR 12.5, 95\% $\mathrm{Cl} 2.7-56.6, \mathrm{p}<0.002$ ), though uncommon. No further risk factors were identified. Conclusion: A history of suicide attempts and the presence of depression are strongly predictive of suicidal behavior in prodromal HD. As these risk factors are among the most robust risk factors for suicide, established suicide risk factors appear applicable to those with prodromal HD.

Copyright $\odot 2011$ S. Karger AG, Basel

\section{Introduction}

Huntington disease (HD) is an autosomal dominant neuropsychiatric disorder caused by an expansion of the HD gene on chromosome 4p16.3 [1] via repetitions of the nucleic acids $C, A$, and $G$ [2]. The condition demonstrates a broad neuropsychiatric phenotype, and commonly presents with disordered behavior, cognition, mood, and motor symptoms $[3,4]$. Since the astute original description of the illness by George Huntington in 1872 [5], suicide has been further connected to the diagnosis of HD.

Much of the literature on suicide in HD backs the antecedent assertion of George Huntington that the illness sometimes conveys a 'tendency' to 'that form of insanity which leads to suicide' [5]. As highlighted in table 1, stud- 
Table 1. Percentage of deaths due to suicide in $\mathrm{HD}$

\begin{tabular}{lllcc}
\hline First author & Location & Time frame & $\begin{array}{c}\mathrm{n} \text { (deceased } \\
\text { with HD) }\end{array}$ & $\begin{array}{c}\text { Deaths due } \\
\text { to suicide, \% }\end{array}$ \\
\hline Reed [7] & Michigan, USA & 1940 & 171 & $7^{\dagger}$ \\
Brothers [15] & Australia & $1953-?$ & 174 & 1.7 \\
Hayden [14] & South Africa & circa 1976 & $328^{\ddagger}$ & 3.4 \\
Chui [16] & Australia & $1952-1979$ & 182 & 1.6 \\
Schoenfeld [11] & New England, USA & $1980-1983$ & 506 & 4.0 \\
Haines [17] & USA* & $1890-1985$ & 253 & 2.0 \\
Farrer [10] & USA* & not reported & 452 & 5.7 \\
Lanska [8] & USA & $1971,1973-1978$ & 3,238 & 0.6 \\
Sorensen [12] & Denmark & $1943-?$ & 395 & 5.6 \\
Di Maio [9] & USA & $1815-1987$ & 2,793 & $7.3^{\S}$ \\
Baliko [6] & Hungary & $1920-1997$ & 396 & 10.1 \\
Alonso [13] & Mexico & $1973-2008$ & 89 & 2.3 \\
\hline
\end{tabular}

This table outlines studies of mortality by suicide in HD. The varied timeframes and locations of these studies hinder any composite figures.

* Data from National Huntington Disease Research Roster, unclear to what if any degree samples overlap.

${ }^{\ddagger}$ Extrapolated from provided data.

${ }^{\dagger}$ Data for non-institutionalized population. No suicides noted in institutionalized population for total percent suicide. Including the larger portion of the sample that was institutionalized, the figure drops to 3.3\%.

${ }^{\S}$ Data reported for HD families. Extrapolating patient data for 90 suicides in known years results in a higher $9 \%$ figure.

ies of suicide in HD have largely focused on the proportions of deaths attributable to suicide [6-17]. These studies have produced quite varied results with suicides comprising between 0.6 and $10.1 \%$ of the deaths in those with HD $[6,8]$. The study by Bailko et al. [6] was conducted in Hungary, where suicide rates are remarkably high, $45 / 100,000$ persons/year per the authors. Barring this study, the upper limit reported appears to be at or near $7 \%$ of deaths due to suicide in HD as reported by two studies [7,9]. The lowest estimate of $0.6 \%$ comes from data obtained through the United States National Center for Health Statistics [8]. Interpretation of the proportion of deaths due to suicide is inherently limited by the background rate of suicide for the study base and the methods by which suicide is ascertained. In two studies that included proportion of suicides for the relevant general population, the proportion of suicides in HD was 2-4 times that expected. Another study found the proportions of deaths due to suicide in HD to be three times that of unaffected siblings and parents and four times that of general population estimates [10]. One study also found higher rates than the general population, though could not calculate a ratio with some suicides occurring in unknown years [9]. In those studies which did not provide data to calculate the proportions of death due to suicide or any direct calculation of rate, some $[18,19]$, but not all [20], studies suggested an association between suicide and HD. The higher risk of suicide observed in HD has not been demonstrated for other dementias, for which most evidence suggests a lower rate of suicide compared to the general population [21].

From general population data, suicide attempts occur with roughly ten times the frequency of completed suicides [22], though there are varied results due to methodological and other study differences [23]. Even when 'unsuccessful,' suicide attempts can result in substantial morbidity [24]. Surveys of individuals living with HD have reported a lifetime history of suicide attempts in $4.8-17.7 \%$, as highlighted in table $2[10,13-15,20,25]$. The lowest rate was seen in the study by Hayden et al. [14], wherein suicide attempts were limited to severe attempts necessitating hospitalization. As demonstrated in the table, most samples of HD demonstrated a prevalence of suicide attempts in the 5-10\% range. The reported frequency of suicide attempts was noted to be higher than general population estimates by Farrer [10]. Comparison of these data to background frequencies in the general population is otherwise challenging. Unweighted estimates from Part II of the National Comorbidity Survey (Part II over-represented those with mental disorders) re- 
Table 2. Lifetime prevalence of suicide attempts for individuals with HD

\begin{tabular}{lllrr}
\hline First author & Location & Time frame & $\mathrm{n}$ & Attempting suicide, \% \\
\hline Brothers [15] & Australia & $1953-?$ & 133 & 5.3 \\
Dewhurst [20] & UK & $1954-?$ & 102 & 9.8 \\
Hayden [14]* & South Africa & circa 1976 & 500 & 4.8 \\
Farrer [10] & USA & not reported & 831 & 17.7 \\
Paulsen [25, 30] & North America, Europe, Australia & $1980-1983$ & 2,835 & 10.3 \\
Alonso [13] & Mexico & $1973-2008$ & 691 & 6.2 \\
\hline
\end{tabular}

This table outlines studies of suicide attempts in HD. The varied time frames, locations, and methods of ascertainment for these studies hinders any composite figures.

* Used threshold of severe attempts necessitating hospitalization.

port a lifetime prevalence of $2.6 \%$ for suicide attempts with intent to die and $4.6 \%$ for attempts regardless of intent [26]. Using identical measures from World Mental Health surveys, lower lifetime prevalences for attempts regardless of intent have been estimated cross-nationally (2.7 vs. $4.6 \%$ ) [27]. Overall, the available data support the notion of individuals with HD being at increased risk of attempting and completing suicide.

Risk factors for completed suicide in HD identified to date include male gender $[6,9-11,14,18]$ and lack of offspring [19]. Akin to the general population literature, suicide attempts in HD have, on the other hand, been associated with female gender [10, 14]. Several papers suggest suicide occurs more frequently early in the course of $\mathrm{HD}$, though data from only one study supports this [11], another refutes [10], and three studies cited to promote this contention do not test this hypothesis [7, 14, 18]. There has been some concern related to the impact of genetic testing for suicide risk [28]. A study of 4,527 test participants found $0.5 \%$ attempted and $0.1 \%$ completed suicide in up to 3 years following testing [29]. Suicidal ideation is most common when patients start experiencing symptoms before receiving a formal diagnosis of HD and in the second stage of HD following diagnosis, when patients become less independent [30]. Similarly, risk of suicide is generally thought to be higher early, but not later in the course of illness for dementias [21], though increased risk of suicidal behavior early in the course of illness has not been established in HD. Given the only crude demographic data available for most studies of suicide in HD, few risk factors apart from gender have been systematically addressed in research to date.

A much greater variety of risk factors for suicide have been identified in general samples [31]. Mental disorders, which are present in over $90 \%$ of suicides, according to psychological autopsy studies, are of particular relevance [32-34]. These include mood disorders, alcohol and drug use disorders, psychotic disorders, personality disorders, eating disorders, and anxiety disorders [35]. Mood and anxiety disorders, in particular, are common in prodromal HD [36]. In a review of the literature by Coryell [37], the most robust predictors of suicide, as identified in several studies, were prior attempts or plan for suicide, male gender, being single or living alone, inpatient psychiatric treatment, and hopelessness. Of these, a history of suicidal plans or behaviors was by far the most replicated [37].

Designed to assess an array of clinical markers and to follow participants prospectively, the PREDICT-HD study provides a unique opportunity to assess a broad array of risk factors for suicidal behavior in prodromal HD. Given the limited literature on suicide risk factors specifically in HD, we sought to assess risk factors for suicidal behavior in prodromal HD using the PREDICT-HD database. We anticipated that suicide attempts and completions would be common and that established risk factors for suicide in the general population would prove relevant to suicide risk in prodromal HD.

\section{Methods}

\section{Sample}

PREDICT-HD is a multi-site longitudinal prospective study designed to identify and track markers of HD prior to the onset of the classic motor symptoms [38], such as chorea and dystonia. Participants were recruited from a total of 32 sites in the USA, Canada, Europe, and Australia. Prodromal HD is defined by the presence of the CAG repeat expansion prior to meeting clinical criteria for the diagnosis of HD. Institutional Review Board approval was obtained at all sites, and participants signed a written informed consent. 
All prodromal HD participants had undergone voluntary independent genetic testing for the CAG repeat expansion prior to entering the PREDICT-HD study, and were aware of their gene status. Those with a CAG repeat expansion of $\geq 36$ served as cases and those who tested negative $(C A G \leq 30)$ were invited to participate as healthy comparisons. Controls were offspring of a parent with HD. Exclusion criteria included the presence of an unstable medical or psychiatric illness, current alcohol or other drug abuse, a history of special education, a history of other central nervous system disease or events, pacemaker or metallic implants, prescription of an antipsychotic in the past 6 months, or phenothiazine-derived antiemetic medication in the past 3 months [38].

Our sample included 735 participants identified as cases prior to formal diagnosis of HD and followed for a median of 3.5 years (mean $=3.7 ; \mathrm{SD}=1.5)$. Another 194 controls were followed for a median of 2.9 years $($ mean $=3.0, \mathrm{SD}=1.4)$. Gene-expanded participants were sub-divided into groups based on an estimated proximity to $\mathrm{HD}$ diagnosis based on current age and CAG repeat length: far ( $>15$ years), mid (9-15 years), and near ( $<9$ years) [39]. Participants were stratified according to proximity to diagnosis because changes in behavior, cognition, emotional regulation, and motor function have been demonstrated prior to HD diagnosis [36, 40-42], and being early in the course of illness has previously been suggested as a risk factor for suicide in HD.

\section{Assessments}

At baseline and during follow-up, information was obtained by trained research staff allowing us to assess a variety of risk factors for suicide including but not limited to hopelessness, separation, death of a loved one, change in sleeping habits, illness/injury, depressed mood, suicidal ideation, and history of suicide attempts. Incarceration and a variety of other life events were also considered. Selected items from the Psychiatric Status Schedule [43], Life Experiences Survey [44], and Unified Huntington's Disease Ratings Scale (UHDRS) [45] were used to identify risk factors for suicidal behavior. The Psychiatric Status Schedule is a standardized interview designed to assess several domains of psychopathology, including depression, anxiety, grandiosity, behavior, and alcohol abuse [43]. The Life Experience Survey assesses both the number of life changes related to work, relationships, and health over the past 12 months and the perception of these changes as positive or negative [44]. Individual items from the Psychiatric Status Schedule and Life Experiences Survey were modeled as potential risk factors. The UHDRS is a clinical rating scale and contained items assessing the frequency and severity of suicidal thoughts [45]. As part of a suicide risk screening measure, a threshold for frequency of suicidal thoughts was set at a score $\geq 2$ or 'sometimes thinking about suicide - at least once a month'. Severity was assessed by an item asking 'Do you have a plan to end it all? Have you taken any steps toward carrying out your plan?' Participants indicating suicidal ideation were evaluated for suicide risk and referred for a clinical evaluation, when appropriate. Participants were administered the self-report Beck Depression Inventory-II (BDI-II) [46, 47], which provided a total score. A separate study-specific suicide risk screening measure used a score of $>13$ to indicate the categorical presence of a depressive syndrome, indicating at least mild depression, which was previously used as a cutoff in samples with neuropsychiatric illness [48].

A total of 103 variables from the above assessments were modeled for their relationship to suicidal behavior: 70 items +2 total scores from the Life Experiences Survey, 14 items + 1 total score from the Psychiatric Status Schedule, 4 items from the UHDRS, total motor score, BDI-II total score, a history of suicide attempts prior to study entry, and 8 items + total score from the study-specific suicide risk assessment metric. The latter extrapolated individual items for suicidal ideation and hopelessness from the BDIII, recorded the presence of a BDI-II $>13$, indicated a Beck Hopelessness Scale [49] score of 9 or higher [50], and used thresholds on questions from the UHDRS on severity and frequency of depression or suicidal ideation.

Suicide attempts were recorded in a reportable event log and identified from unscripted screenings at each annual visit and 6 -month phone call. The suicide completion was verified by autopsy report. To maximize statistical power, the clinically relevant outcomes of suicide attempts and completions were combined into a single variable, suicidal behavior, akin to prior studies [51-53].

\section{Data Analysis}

Descriptive statistics for the sample were compiled to compare differences between those with and without suicidal behavior during follow-up; t tests were used to assess differences in continuous variables, and $\chi^{2}$ analyses were used for categorical variables.

The primary outcome of time to first suicidal behavior was assessed in survival analysis using Cox proportional hazards regression. The 103 variables from the aforementioned scales were assessed in univariate models as time-dependent covariates. A history of suicide attempts was assessed at baseline and subsequently modeled as a time-independent covariate. All statistical tests were corrected for multiple comparisons using a false discovery rate $(F D R)$ of $\leq 0.05$ [54]. Individuals who did not demonstrate suicidal behavior were censored at last visit or when lost to follow-up. Multivariate Cox models were assembled to assess for any independence to the above findings. These analyses assumed proportional hazards and that censoring and suicidal behavior were independent. Unless otherwise noted, statistical tests used a two-tailed $\alpha$ of 0.05. Analyses were performed using SAS 9.2.

\section{Results}

The sociodemographic and clinical characteristics of the entire sample are detailed in table 3 . A total of 735 individuals with prodromal HD and a mean age of 41.2 years were included in the sample, of which $63.3 \%$ were female. Proximity to diagnosis was estimated as $37.8 \%$ far ( $>15$ years), $37.8 \%$ mid ( $9-15$ years), and $24.4 \%$ near $(<9$ years) to diagnosis but not diagnosed. Most participants who demonstrated suicidal behavior were female (Fisher's exact test $\mathrm{p}=0.04)$. Other sociodemographic and clinical variables (table 3) did not differ between those with and without suicidal behavior. Those with suicidal behavior had higher scores on the BDI-II at the prior visit compared with those who did not engage in suicidal behavior (16.3 vs. $7.6, t=-3.53$, d.f. $=730, p=0.0004$ ). Cases appeared similar to controls with regard to gender 
Table 3. Clinical and sociodemographic characteristics of sample

\begin{tabular}{|c|c|c|c|c|}
\hline & $\begin{array}{l}\text { Suicidal behavior } \\
(\mathrm{n}=13)\end{array}$ & $\begin{array}{l}\text { No suicidal behavior } \\
(\mathrm{n}=722)\end{array}$ & $\begin{array}{l}\text { Prodromal HD cases } \\
(\mathrm{n}=735)\end{array}$ & $\begin{array}{l}\text { Controls } \\
(\mathrm{n}=194)\end{array}$ \\
\hline Age at intake, years & $38.9 \pm 5.0$ & $41.2 \pm 9.8$ & $41.2 \pm 9.7$ & $43.7 \pm 11.0$ \\
\hline Years of education & $14.6 \pm 3.5$ & $14.4 \pm 2.7$ & $14.4 \pm 2.7$ & $14.7 \pm 2.7$ \\
\hline CAG repeats $^{\dagger}$ & $42.5 \pm 1.5$ & $42.4 \pm 2.6$ & $42.4 \pm 2.5$ & \\
\hline Females & $12(92.3)$ & $453(62.7)$ & $465(63.3)$ & $128(66.0)$ \\
\hline \multicolumn{5}{|c|}{ Proximity to diagnosis ${ }^{\dagger, *}$} \\
\hline Far & $5(38.5)$ & $271(37.7)$ & $276(37.8)$ & \\
\hline Mid & $6(46.1)$ & $270(37.6)$ & $276(37.8)$ & \\
\hline Near & $2(15.4)$ & $177(24.7)$ & $179(24.4)$ & \\
\hline
\end{tabular}

and education. though controls were significantly older than cases $(t=2.92$, d.f. $=277.84, p=0.004)$.

Twelve participants (1.6\%) attempted suicide and 1 participant completed suicide. One participant had 2 attempts. Thus, a total of 13 participants demonstrated suicidal behavior as operationally defined, and a total of 14 events were observed. Suicidal behavior did not significantly differ with estimated proximity to diagnosis. Based on a total of 2,718 person-years of follow-up, we observed 4.8 suicide attempts per 1,000 person-years among individuals with prodromal HD. Twelve of the 13 demonstrating suicidal behavior were female. No suicidal behavior was observed in the 194 controls, precluding modeling in survival analysis. On cross-tabulation, the frequency of suicidal behaviors did not significantly differ between cases and controls ( $\mathrm{p}=0.08$ by Fisher's exact test).

Risk factors for suicidal behavior were modeled in Cox regression. In univariate analyses, a history of suicide attempts (HR 8.5, 95\% CI 2.8-26.1, p < 0.0002, FDR $=0.02$ ) and a BDI-II $>13$ (HR 7.2, 95\% CI 2.3-22.0, p $<0.0006$, $\mathrm{FDR}=0.02$ ) were associated with suicidal behavior. Compared to those without suicidal behavior, $38 \%$ (vs. $7 \%$ ) of those with suicidal behavior had a history of suicide attempts and $62 \%$ (vs. $18 \%$ ) had a BDI-II $>13$ on the visit before attempt or censoring. The total score of the BDI-II was also associated with suicidal behavior after controlling for multiple comparisons (HR 1.07, 95\% CI 1.03-1.11, p < 0.001 , FDR $=0.02$ ). Incarceration in the past 2 years was also significantly associated with suicidal behavior (HR $12.5,95 \%$ CI 2.7-56.6, $\mathrm{p}<0.002, \mathrm{FDR}=0.03$ ) though occurred infrequently overall and was present in only 2 of the cases with suicidal behavior and 33 of the cases without.
Table 4. Multivariate Cox regression models of risk factors for suicidal behavior

\begin{tabular}{|c|c|c|}
\hline & HR (95\% CI) & $\mathrm{p}$ value \\
\hline \multicolumn{3}{|l|}{ Model 1} \\
\hline Depression $^{\dagger}$ & $5.5(1.8-17.5)$ & 0.004 \\
\hline Prior attempts & $5.8(1.8-18.4)$ & 0.003 \\
\hline \multicolumn{3}{|l|}{ Model 2} \\
\hline Depression $^{\dagger}$ & $6.3(2.0-19.7)$ & 0.002 \\
\hline Incarceration past 2 years & $6.9(1.5-32.7)$ & 0.01 \\
\hline \multicolumn{3}{|l|}{ Model 3} \\
\hline Incarceration past 2 years & $6.9(1.4-33.3)$ & 0.02 \\
\hline Prior attempts ${ }^{\ddagger}$ & $6.9(2.2-22.3)$ & 0.001 \\
\hline
\end{tabular}

${ }^{\dagger}$ Defined as a score of $>13$ on the BDI II.

* Assessed at baseline.

Because 13 events were observed, no more than 2 variables were included simultaneously in multivariate models, which included variables for prior incarceration, prior suicide attempts, and a BDI-II $>13$. The results of these bivariate models are detailed in table 4 , which shows the observed associations appeared independent of each other.

\section{Discussion}

Our results demonstrate that the presence of depression and a history of suicide attempts are relevant risk factors for suicidal behavior in prodromal HD. These are 
among the most robust risk factors for depression reported in the medical literature [37], lending support to the practice of assessing suicide risk in this population as individuals rather than distinctly as individuals with prodromal HD. Our data did not demonstrate a significantly increased risk of suicidal behavior in cases compared to controls, though this was a highly selected sample and no incidents of suicidal behavior were observed in the smaller control group. Based on the studies reviewed in the introduction, HD may itself be considered a risk factor for suicide. The infrequently observed event of prior incarceration was also associated with suicide. This finding was unexpected and begs the question of whether any association reflects the stressful event itself or factors that predispose to incarceration, such as impulsivity [55-57]. A specific measure of impulsivity was not available, though one has recently been added in a revised PREDICT-HD protocol.

Our study has several important limitations. Suicide attempts and completions were combined into a single variable, representing suicidal behavior. Those who attempt suicide represent a similar, but not identical, group to those who complete suicide [58]. Our findings, particularly with regard to gender, may not be generalizable to the assessment of risk for completed suicide as risk factors for suicide attempts do not always parallel those of suicide completions $[59,60]$. Nonetheless, suicide attempts represent a clinically relevant outcome and the prediction of suicidal behavior an important and useful clinical outcome. Only 13 individuals demonstrated suicidal behavior in our sample. This limits both our power to assess risk factors and ability to reliably include more than two risk factors in a single regression model. Thus, previously established risk factors for suicide, not found to be associated with suicidal behavior in this sample, should not be assumed irrelevant to the assessment of suicide risk in HD. While the nature of the study makes ascertainment of suicide quite certain, it is possible that deaths due to other causes, such as accidents, represented suicides. Further, the sensitivity of our methods for ascertaining suicide attempts is unknown and suicide attempts may be underreported. This limitation, however, is pervasive throughout the epidemiological literature on suicide attempts. Regardless, our estimates of suicide and suicide attempts may be underestimated and would best be considered conservative lower limits. Suicidal behavior was indeed observed less frequently than perhaps expected in this select sample of patients with prodromal $\mathrm{HD}$, in which participants with unstable psychiatric illness or receiving treatment with antipsychotics were excluded. Against a backdrop of studies reporting increased risk of suicide in HD, our findings beg some explanation. Rates could be lower due to selection bias, as this sample includes those who have had gene testing and agreed to participate in an intensive long-term study. Risk may also be lower for individuals with prodromal HD compared to those with HD. It is also possible that as a result of participating in the study, individuals at risk were appropriately referred to clinical resources, mitigating this risk. Further, the very act of participating in the study may provide an understanding support network. Thus, results related to overall risk of suicidal behavior may not generalize to the population at large with HD. When including retrospective data, however, 53 participants $(7.2 \%)$ had a suicide attempt prior to participating or prospectively observed during participation. This figure is higher than that of cross-national lifetime estimates of $2.7 \%$ [27] and akin to that of the aforementioned prevalences of lifetime suicide attempts with HD as reported in the reviewed literature.

Prospective follow-up of a large sample and rigorous clinical assessments are among the most notable strengths of our study. Prior studies of suicide in HD have been largely limited to registry studies or national death indices. The PREDICT-HD data facilitated the assessment of a variety of potentially relevant risk factors to determine the risk of suicidal behavior in survival analyses, the optimal method of analyzing this time to event data. With multiple assessments, our analyses were additionally able to assess risk factors as time-varying covariates. Our results were able to uniquely contribute to the literature by identifying several risk factors for suicide in prodromal HD, many of which could not be assessed by studies published to date, which primarily assessed readily available demographic variables such as gender.

There is little reason to assume the risk factors identified do not generalize to the population at large with HD, as these risk factors are among the most robust risk factors from studies in other populations. While it is quite probable that risk of suicide observed in this sample differs from the study base (of all individuals at risk for HD) from which the cases arose, it seems implausible that the risk factors themselves would not generalize. These data suggest suicide risk assessment strategies for individuals with HD may be employed with similar methods and consideration of risk factors. While there was a tendency for more suicide attempts among those earlier in the course of illness, based on estimated time to diagnosis, any differences were not statistically significant, and our sample was limited to individuals with prodromal HD. 
There was further no association between total motor score on the UHDRS and suicidal behavior. The available literature does not convincingly support consideration of 'early in the course of illness' as an established risk factor for suicide in HD, and further study is needed related to suicide risk by illness severity. Some speculate that loss of insight over the course of illness may reduce suicide risk [21]. Continued follow-up of this cohort will provide an important opportunity to answer this question.

Individuals with prodromal HD face an elevated risk of suicide, yet clinicians working with this at-risk population have limited data upon which to assess suicide risk. Our results suggest that standard assessments for suicide risk may be reasonably applied to populations with prodromal HD and presumably HD with depression and a history of suicide attempts - two of the most established predictors in the suicide literature - as predictors of suicidal behavior in prodromal HD. The risk factor of depression is particularly noteworthy as it represents a po- tentially modifiable risk factor for suicide. Although randomized controlled trials for major depression in prodromal HD or HD are lacking [61], depression may be more common in HD and is generally treatable [25]. This underscores the importance of screening for depressive syndromes in individuals with prodromal HD or HD.

\section{Acknowledgments}

J.S.P. and the PREDICT-HD study is supported by the NIH (NS40068) and CHDI Foundation, Inc. J.G.F. is supported by the CHDI Foundation, Inc., the NIH (1K23MH083695-01A210), NARSAD, the Nellie Ball Trust Research Fund, and the Institute for Clinical and Translational Science at The University of Iowa (3 UL1 RR024979-03S4). J.G.F. currently serves on colleagues' studies of HD with Neurosearch and the NIH.

We thank the PREDICT-HD sites, the study participants, and the National Research Roster for Huntington Disease Patients and Families. A full list of PREDICT-HD investigators, coordinators, motor raters, and cognitive raters can be found online.

\section{References}

1 Landegent JE, Jansen in de Wal N, FisserGroen YM, Bakker E, van der Ploeg M, Pearson PL: Fine mapping of the Huntington disease linked D4S10 locus by non-radioactive in situ hybridization. Hum Genet 1986;73: 354-357.

-2 The Huntington's Disease Collaborative Research Group: A novel gene containing a trinucleotide repeat that is expanded and unstable on Huntington's disease chromosomes. Cell 1993;72:971-983.

-3 Myers RH: Huntington's disease genetics. NeuroRx 2004;1:255-262.

4 Walker FO: Huntington's disease. Lancet 2007;369:218-228.

5 Huntington G: On chorea: George Huntington, M.D. J Neuropsychiatry Clin Neurosci 2003; 15:109-112.

6 Baliko L, Csala B, Czopf J: Suicide in Hungarian Huntington's disease patients. Neuroepidemiology 2004;23:258-260.

7 Reed TE, Chandler JH: Huntington's chorea in Michigan. I. Demography and genetics. Am J Hum Genet 1958;10:201-225.

-8 Lanska DJ, Lavine L, Lanska MJ, Schoenberg BS: Huntington's disease mortality in the United States. Neurology 1988;38:769-772.

$\checkmark 9$ Di Maio L, Squitieri F, Napolitano G, Campanella G, Trofatter JA, Conneally PM: Suicide risk in Huntington's disease. J Med Genet 1993;30:293-295.
10 Farrer LA: Suicide and attempted suicide in Huntington disease: implications for preclinical testing of persons at risk. Am J Med Genet 1986;24:305-311.

11 Schoenfeld M, Myers RH, Cupples LA, Berkman B, Sax DS, Clark E: Increased rate of suicide among patients with Huntington's disease. J Neurol Neurosurg Psychiatry 1984;47: 1283-1287.

12 Sorensen SA, Fenger K: Causes of death in patients with Huntington's disease and in unaffected first degree relatives. J Med Genet 1992;29:911-914.

13 Alonso ME, Ochoa A, Boll MC, Sosa AL, Yescas P, Lopez M, Macias R, Familiar I, Rasmussen A: Clinical and genetic characteristics of Mexican Huntington's disease patients. Mov Disord 2009;24:2012-2015.

14 Hayden MR, Ehrlich R, Parker H, Ferera SJ: Social perspectives in Huntington's chorea. S Afr Med J 1980;58:201-203.

15 Brothers CR: Huntington's chorea in Victoria and Tasmania. J Neurol Sci 1964;11:405420.

16 Chiu E, Alexander L: Causes of death in Huntington's disease. Med J Aust 1982;1:153.

17 Haines JL, Conneally PM: Causes of death in Huntington disease as reported on death certificates. Genet Epidemiol 1986;3:417423.

18 Bolt JM: Huntington's chorea in the West of Scotland. Br J Psychiatry 1970;116:259-270.
19 Lipe H, Schultz A, Bird TD: Risk factors for suicide in Huntingtons disease: a retrospective case controlled study. Am J Med Genet 1993;48:231-233

20 Dewhurst K, Oliver JE, McKnight AL: Sociopsychiatric consequences of Huntington's disease. Br J Psychiatry 1970;116:255-258.

21 Haw C, Harwood D, Hawton K: Dementia and suicidal behavior: a review of the literature. Int Psychogeriatr 2009;21:440-453.

22 Hawton K, Arensman E, Wasserman D, Hulten A, Bille-Brahe U, Bjerke T, Crepet $P$, Deisenhammer E, Kerkhof A, De Leo D, Michel K, Ostamo A, Philippe A, Querejeta I, Salander-Renberg E, Schmidtke A, Temesvary $B$ : Relation between attempted suicide and suicide rates among young people in $\mathrm{Eu}$ rope. J Epidemiol Community Health 1998; 52:191-194.

23 Welch SS: A review of the literature on the epidemiology of parasuicide in the general population. Psychiatr Serv 2001;52:368-375.

24 Dubovsky SL, Dubovsky AN: Mood disorders and the outcome of suicidal thoughts and attempts. Crit Care Clin 2008;24:857874, ix.

25 Paulsen JS, Nehl C, Hoth KF, Kanz JE, Benjamin M, Conybeare R, McDowell B, Turner B: Depression and stages of Huntington's disease. J Neuropsychiatry Clin Neurosci 2005; 17:496-502. 
-26 Nock MK, Kessler RC: Prevalence of and risk factors for suicide attempts versus suicide gestures: analysis of the National Comorbidity Survey. J Abnorm Psychol 2006;115:616623.

-27 Nock MK, Borges G, Bromet EJ, Alonso J, Angermeyer M, Beautrais A, Bruffaerts R, Chiu WT, de Girolamo G, Gluzman S, de Graaf R, Gureje O, Haro JM, Huang Y, Karam E, Kessler RC, Lepine JP, Levinson D, Medina-Mora ME, Ono Y, Posada-Villa J, Williams D: Cross-national prevalence and risk factors for suicidal ideation, plans and attempts. Br J Psychiatry 2008;192:98-105.

28 Robins Wahlin TB: To know or not to know: a review of behaviour and suicidal ideation in preclinical Huntington's disease. Patient Educ Couns 2007;65:279-287.

-29 Almqvist EW, Bloch M, Brinkman R, Craufurd D, Hayden MR: A worldwide assessment of the frequency of suicide, suicide attempts, or psychiatric hospitalization after predictive testing for Huntington disease. Am J Hum Genet 1999;64:1293-1304.

- 30 Paulsen JS, Hoth KF, Nehl C, Stierman L: Critical periods of suicide risk in Huntington's disease. Am J Psychiatry 2005; 162:725731.

31 Fiedorowicz JG, Weldon K, Bergus G: Determining suicide risk (Hint: A screen is not enough). J Fam Pract 2010;59:256-260.

- 32 Foster T, Gillespie K, McClelland R: Mental disorders and suicide in Northern Ireland. Br J Psychiatry 1997;170:447-452.

- 33 Henriksson MM, Aro HM, Marttunen MJ, Heikkinen ME, Isometsa ET, Kuoppasalmi KI, Lonnqvist JK: Mental disorders and comorbidity in suicide. Am J Psychiatry 1993; 150:935-940.

-34 Rich CL, Young D, Fowler RC: San Diego suicide study. I. Young vs old subjects. Arch Gen Psychiatry 1986;43:577-582.

35 Fiedorowicz JG, Black DW, Coryell WH: Suicide and Mental Morbidity; in Shrivastava A (ed): Handbook of Suicide Behaviour. London, Royal College of Psychiatrists, in press.

- 36 Duff K, Paulsen JS, Beglinger LJ, Langbehn DR, Stout JC: Psychiatric symptoms in Huntington's disease before diagnosis: the PREDICT-HD study. Biol Psychiatry 2007;62: 1341-1346.

-37 Coryell WH: Clinical assessment of suicide risk in depressive disorder. CNS Spectr 2006; 11:455-461.
38 Paulsen JS, Hayden M, Stout JC, Langbehn DR, Aylward E, Ross CA, Guttman M, Nance M, Kieburtz K, Oakes D, Shoulson I, Kayson E, Johnson S, Penziner E: Preparing for preventive clinical trials: the PREDICT-HD study. Arch Neurol 2006;63:883-890.

- 39 Langbehn DR, Brinkman RR, Falush D, Paulsen JS, Hayden MR: A new model for prediction of the age of onset and penetrance for Huntington's disease based on CAG length. Clin Genet 2004;65:267-277.

40 Robins Wahlin TB, Lundin A, Dear K: Early cognitive deficits in Swedish gene carriers of Huntington's disease. Neuropsychology 2007;21:31-44.

41 Solomon AC, Stout JC, Weaver M, Queller S, Tomusk A, Whitlock KB, Hui SL, Marshall J, Jackson JG, Siemers ER, Beristain X, Wojcieszek J, Foroud T: Ten-year rate of longitudinal change in neurocognitive and motor function in prediagnosis Huntington disease. Mov Disord 2008;23:1830-1836.

- 42 Biglan KM, Ross CA, Langbehn DR, Aylward EH, Stout JC, Queller S, Carlozzi NE, Duff K, Beglinger LJ, Paulsen JS: Motor abnormalities in premanifest persons with Huntington's disease: the PREDICT-HD study. Mov Disord 2009;24:1763-1772.

43 Spitzer RL, Endicott J, Fleiss JL, Cohen J: The psychiatric status schedule: a technique for evaluating psychopathology and impairment in role functioning. Arch Gen Psychiatry 1970;23:41-55.

44 Sarason IG, Johnson JH, Siegel JM: Assessing the impact of life changes: development of the Life Experiences Survey. J Consult Clin Psychol 1978;46:932-946.

45 Huntington Study Group: Unified Hunting ton's Disease Rating Scale: reliability and consistency. Mov Disord 1996;11:136-142.

46 Beck AT, Steer RA, Brown GK: Manual for the Beck Depression Inventory-II. San Antonio, Psychological Corporation, 1996.

47 Beck AT, Steer RA, Ball R, Ranieri W: Comparison of Beck Depression Inventories -IA and -II in psychiatric outpatients. J Pers Assess 1996;67:588-597.

48 Inoue T, Kitagawa M, Tanaka T, Nakagawa S, Koyama T: Depression and major depressive disorder in patients with Parkinson's disease. Mov Disord 2010;25:44-49.

49 Beck AT, Weissman A, Lester D, Trexler L: The measurement of pessimism: the hopelessness scale. J Consult Clin Psychol 1974; 42:861-865.

-50 Beck AT, Steer RA, Kovacs M, Garrison B: Hopelessness and eventual suicide: a 10-year prospective study of patients hospitalized with suicidal ideation. Am J Psychiatry 1985; 142:559-563.
1 Fiedorowicz JG, Leon AC, Keller MB, Solomon DA, Rice JP, Coryell WH: Do risk factors for suicidal behavior differ by affective disorder polarity? Psychol Med 2009;39: 763-771.

-52 Brent DA, Bridge J, Johnson BA, Connolly J: Suicidal behavior runs in families: a controlled family study of adolescent suicide victims. Arch Gen Psychiatry 1996;53:11451152.

53 Marangell LB, Bauer MS, Dennehy EB, Wisniewski SR, Allen MH, Miklowitz DJ, Oquendo MA, Frank E, Perlis RH, Martinez JM, Fagiolini A, Otto MW, Chessick CA, Zboyan HA, Miyahara S, Sachs G, Thase ME: Prospective predictors of suicide and suicide attempts in 1,556 patients with bipolar disorders followed for up to 2 years. Bipolar Disord 2006;8:566-575.

54 Benjamini Y, Hochberg Y: Controlling the false discovery rate: a practical and powerful approach to multiple testing. J R Stat Soc Series B (Methodol) 1995;57:289-300.

55 DeJong J, Virkkunen M, Linnoila M: Factors associated with recidivism in a criminal population. J Nerv Ment Dis 1992;180:543550.

56 Cherpitel CJ, Tam TW: Variables associated with DUI offender status among whites and Mexican Americans. J Stud Alcohol 2000;61: 698-703.

57 Babinski LM, Hartsough CS, Lambert NM: Childhood conduct problems, hyperactivity-impulsivity, and inattention as predictors of adult criminal activity. J Child Psychol Psychiatry 1999;40:347-355.

58 Beautrais AL: Suicide and serious suicide attempts in youth: a multiple-group comparison study. Am J Psychiatry 2003;160:10931099.

59 Fiedorowicz JG, Coryell WH: Cholesterol and suicide attempts: a prospective study of depressed inpatients. Psychiatry Res 2007; 152:11-20.

60 Kuo WH, Gallo JJ, Tien AY: Incidence of suicide ideation and attempts in adults: the 13year follow-up of a community sample in Baltimore, Maryland. Psychol Med 2001;31: 1181-1191.

61 Holl AK, Wilkinson L, Painold A, Holl EM, Bonelli RM: Combating depression in Huntington's disease: effective antidepressive treatment with venlafaxine XR. Int Clin Psychopharmacol 2010;25:46-50. 